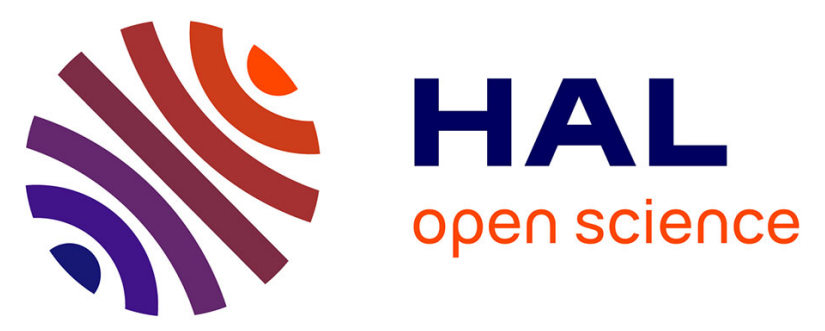

\title{
High accuracy measurements of the intrapixel sensitivity of VIS to LWIR astronomical detectors: experimental demonstration
}

\author{
T. Viale, C. Ketchazo, N. Guerineau, O. Boulade, F. de La Barrière, V. \\ Moreau, L. Mugnier, D. Dubreuil, A. Bonnefois, C. Delisle, et al.
}

\section{To cite this version:}

T. Viale, C. Ketchazo, N. Guerineau, O. Boulade, F. de La Barrière, et al.. High accuracy measurements of the intrapixel sensitivity of VIS to LWIR astronomical detectors: experimental demonstration. SPIE 2016 Astronomical Telescopes + Instrumentation Session Adaptive Optics Systems, Jun 2016, EDIMBOURG, United Kingdom. hal-01354125

\section{HAL Id: hal-01354125 \\ https://hal.science/hal-01354125}

Submitted on 17 Aug 2016

HAL is a multi-disciplinary open access archive for the deposit and dissemination of scientific research documents, whether they are published or not. The documents may come from teaching and research institutions in France or abroad, or from public or private research centers.
L'archive ouverte pluridisciplinaire HAL, est destinée au dépôt et à la diffusion de documents scientifiques de niveau recherche, publiés ou non, émanant des établissements d'enseignement et de recherche français ou étrangers, des laboratoires publics ou privés. 


COMMUNICATION A CONGRES
High accuracy measurements of the
intrapixel sensitivity of VIS to LWIR
astronomical detectors :
experimental demonstration
T. Viale (ONERA), C. Ketchazo, N. Guerineau
(ONERA), O. Boulade, F. De La Barrière (ONERA),
V. Moreau, L. Mugnier (ONERA), D. Dubreuil,
A. Bonnefois (ONERA), C. Delisle, G. Druart (ONERA),
P. Mulet
SPIE 2016 Astronomical Telescopes + Instrumentation
Session Adaptive Optics Systems
EDIMBOURG, GRANDE-BRETAGNE
26 juin-1 juillet 2016
TP 2016-445

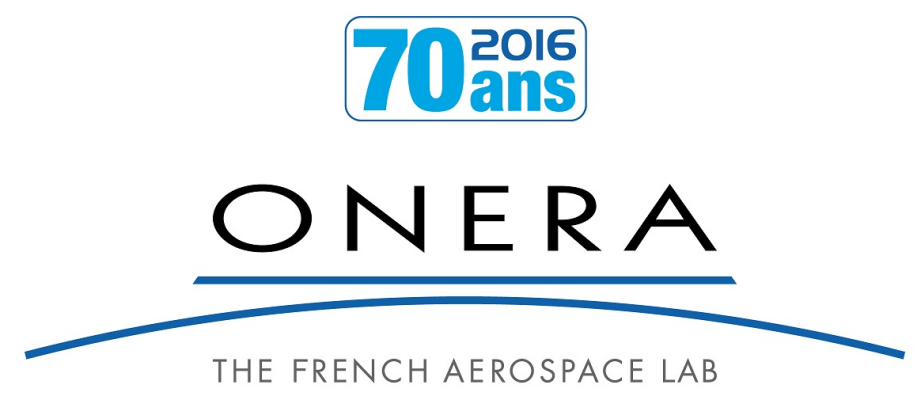




\title{
High accuracy measurements of the intrapixel sensitivity of VIS to LWIR astronomical detectors : experimental demonstration
}

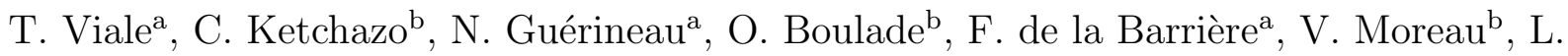

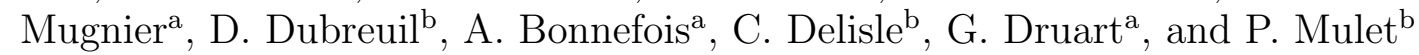 \\ ${ }^{a}$ ONERA, the French Aerospace Lab, F-91761 Palaiseau, France \\ bervice D'Astrophysique, CEA-Saclay, Orme des Merisiers, Gif-sur-Yvette 91191, France
}

\begin{abstract}
The reduction of systematic effects is necessary to improve the accuracy in imaging and astrometry. For example, in Euclid Mission which aims at carrying out accurate measurements of dark energy and quantifying precisely its role in the evolution of the Universe, systematic effects need at be controlled to a level better than $10^{-7}$ (Euclid, Science Book). To achieve this goal, a high-level of knowledge of the system point spread function (PSF) is required. This paper follows the concept-paper presented at the last SPIE conference ${ }^{1}$ and gives the recent developments achieved in the design of the test bench for the intrapixel sensitivity measurements. The measurement technique we use is based on the projection of a high spatial resolution periodic pattern on the detector using the self-imaging property of a new class of diffractive objects named continuously self-imaging gratings (CSIG) and developed at ONERA. The principle combines the potential of global techniques, which make measurements at once on the whole FPA, and the accuracy of spot-scan-based techniques, which provide high local precision.
\end{abstract}

Keywords: Intrapixel characterization, Infrared detector, Point Spread Function, CSIG, Bayesian approach, EUCLID, Astronomy, Talbot effect

\section{INTRODUCTION}

Measuring the intrapixel sensitivity of a detector has become an unavoidable step for the characterization of high precision systems in space imagery. Indeed, this operation is of great interest : on the one hand, the technologist could check the quality of the pixels and study phenomena related to detection, such as scattering or cross-talk between adjacent pixels, and on the other hand, the optical designer could evaluate the impact of the spatial filtering of the pixels on image quality. Nowadays, to achieve wide field of view at an affordable cost, many instruments designed for space applications operate in an under-sampled mode. In this condition, the sub-pixel sensitivity variations can have an important effect, ${ }^{2}$ to obtain good imagery and photometry. Scientific purpose of ESA's EUCLID space mission is to achieve wide-field imaging and accurate photometry. ${ }^{3}$ The satellite is designed to carry out both weak lensing and galaxy clustering cosmological measurements, using a payload comprising a visible imager (VIS) and a near-infrared spectrograph-imager (NIR). Both instruments VIS and NIR cover a field of view of $0.5^{\circ}$ and are under-sampled because each FPA has a limited number of pixels. Then these instruments could be possibly affected by the IPSV (Intra-Pixel Sensitivity Variations). That is why the IPSV must be precisely evaluated and corrected (if necessary) in the Euclid final images.

Since many years ONERA has developed for many years a global characterization method based on the Talbot effect. ${ }^{4}$ The principle consists in projecting a high-resolution pattern onto the detector, using the self-imaging property (also called Talbot effect) of a specific grating called a CSIG (Continously Self-Imaging Grating). The pattern, as an interferogram, excites a precise number of spatial frequencies in the Fourier domain allowing to determine the MTF of the detection system. ${ }^{5}$ However, this technique is not sufficient enough to explore the pixel response at a sampling pitch inferior to the pixel size. Many intrapixel characterization techniques are used currently, such as the spot scan notably for EUCLID mission ${ }^{6} .^{7}$ Nevertheless, this technique implies the hypothesis of a uniform response on the whole focal place array (FPA). Indeed, due to the temporal cost of this 
technique, it would be impossible to characterize the entire FPA, that is why only few pixels are spot-scanned. In this paper, we present a new characterization method which combines the advantages of both the global CSIG technique and the spot scan.

The concept consists in projecting an interferogram on the whole surface of the detector. This interferogram, as a self-image of the CSIG, is designed to explore the pixel's EUCLID CCD (pitch $12 \mu \mathrm{m}$ ) MTF to $500 \mathrm{~mm}^{-1}$, which corresponds to explore the pixel response with an accuracy of $1 \mu \mathrm{m}$ (or a twelfth of pixel). ${ }^{1}$ Nevertheless, the pixel pitch under-samples the interferogram, cutting the MTF to a spatial frequency of $83 \mathrm{~mm}^{-1}$, it is then necessary to microscan the CSIG to over-sample the interferogram. This microscan step consists in moving the CSIG at a pitch of $1 \mu \mathrm{m}$ to ensure that a pixel - or a restrained zone of pixels on the detector - observes a complete period of the interferogram. At each microscan position, an under-sampled image is acquired, and finally the whole set of images is combined in order to obtain an interferogram correctly sampled. A deconvolution software is then used to reconstruct the pixel response with an accuracy of $1 \mu \mathrm{m}$.

In order to respond to the EUCLID consortium requirements, this high accuracy pixel response has to be estimated with an error inferior to $10 \%$ ! This implies a lot of constraints on the test bench components accuracy, with the establishment of an error budget associated to each component.

We have developed a first demonstration test bench to study the main issues of this new technique in order to build the final test bench called INTRAPIX for the EUCLID mission.

In the following paragraphs, we will present the experimental demonstrator test bench with the deconvolution software, the error budget and the estimation of the reconstruction error associated to our test bench components. Finally, the first experimental results and the ongoing developments will be presented on part 4 and 5 .

\section{GENERAL DESCRIPTION OF THE EXPERIMENT}

A detailed description of the test bench has been presented in a precedent paper. ${ }^{1}$ In this section we will describe the principal characteristics of the components, and the principle of the inversion software.

\subsection{Methodology of measurement}

The purpose of the demonstrator is to combine the advantages of the spot scan technique with the capability of the CSIG technique to characterize the whole detector surface at a subpixel accuracy.

\subsection{Description of the test bench}

This test bench has been designed to be able to measure a detector pixel response to a twelfth pixel accuracy minimum. The reference parameter we used is the pixel point spread function (PSF) for each pixel, or for a restrained zone of the detector. The global technique developed by ONERA allows a measurement of the mean response for the entire surface of the focal plane array, but it is impossible to explore space frequencies high enough to determine the PSF at a sufficient accuracy. To reach this purpose, a microscan step has been added to the experiment. The idead is to place the CSIG on a two-axis micrometric translation system and scan the CSIG pattern in front of each pixel. It is so possible to explore the pixel response to an accuracy of $1 \mu \mathrm{m}$.

The test bench is composed of a white source connected to an optical fiber placed at a collimator entrance. The parallel beam out of the collimator illuminates the CSIG mounted on the microscan system. The formed interferogram is then projected on the tested detector. For our demonstrator, the detector is a $1024^{*} 10241 \mathrm{M} 60$ DALSA camera CCD with a $12 \mu m$ pixel pitch.

Obtaining an information to the twelfth of pixel is equivalent to exploring the detector MTF up to 12 times the detector Nyquist frequency. The Nyquist frequency is given by the equation :

$$
f_{N y}=1 /\left(2 p_{p i x}\right)
$$


with $p_{p i x}$ the detector pixel pitch equal to $12 \mu \mathrm{m}$, which can be traduced as a spatial frequency of $500 \mathrm{~mm}^{-1}$ :

$$
12 \times f_{N y}=12 \times \frac{1}{24}=\frac{1}{2} \mu m^{-1}=500 \mathrm{~mm}^{-1}
$$
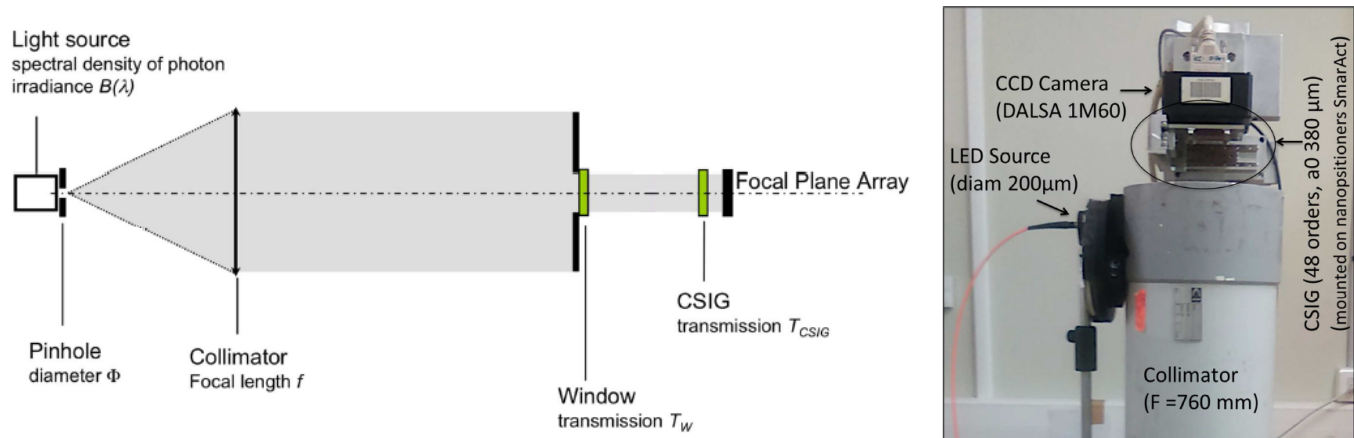

Figure 1. On the left : Schematic view of the test bench based on a continuously self-imaging grating (CSIG). On the right : experimental set-up

\subsubsection{The pinhole}

The pinhole has a filtering effect on the interferogram projected on the detector. The cut-off frequency given by the pinhole diameter is given by :

$$
\rho_{c, \text { pinhole }}=1.22 /\left(\Phi^{\prime}\right)
$$

with $\Phi^{\prime}$ the pinhole diameter in the image plane given by :

$$
\Phi^{\prime}=\Phi \frac{d}{f}
$$

with $\Phi$ the pinhole diameter, $d$ the distance between the CSIG and the detector, and $f$ the collimator's focal length. It is necessary to dimension this pinhole in order to allow an exploration beyond 12 times the Nyquist frequency. In our test bench, $f$ is equal to $760 \mathrm{~mm}$, and $d$ can't be less than $8 \mathrm{~mm}$ because of the test bench constraints. We chosse a pinhole diameter equal to $200 \mu \mathrm{m}$. In this way, $\rho_{c, \text { pinhole }}=579 \mathrm{~mm}^{-1}$, which exceeds the spatial frequency of $500 \mathrm{~mm}^{-1}$ to be measured, ie. $\rho_{c, p i n h o l e}$ has to be superior to the spatial frequency of $500 \mathrm{~mm}^{-1}$ to be measured.

\subsubsection{The CSIG}

The CSIG has to be dimensionned specifically. Indeed, the interferogram produced by the CSIG has to be able to excite a sufficient number of spatial frequencies to explore the pixel MTF to $500 \mathrm{~mm}^{-1}$. A detailled description of the CSIGs and their properties can be found in. ${ }^{5}$ We will present here the fundamental notions allowing to describe the CSIG dimensionning. As a diffraction grating, the CSIG presents a periodic pattern, with a lacunar spatial-frequency spectrum. These spatial frequencies are contained in a circle of radius $\nu_{c}$ given by :

$$
\nu_{c}=2 \frac{\eta}{a_{0}}
$$

with $\nu_{c}$ the CSIG cut-off frequency, $a_{0}$ the period of the CSIG, and $\eta$ a dimensionless scaling factor corresponding to the interferogram central spot diameter. ${ }^{1}$ When varying $\eta$, the number of orders diffracted by the CSIG changes. For a $N$ order CSIG, the number $N^{\prime}$ of spatial frequencies of the CSIG's optical transfert function $(\mathrm{OTF})$ is given $\mathrm{by}^{8}$ : 


$$
N^{\prime}=\frac{N^{2}}{2}+1
$$

Moreover, to correctly sample this OTF, the minimum number of spatial frequencies to excite $N^{\prime}$ is given by $^{1}$ :

$$
N_{\min }^{\prime}=\pi\left(\frac{\nu_{c}}{b}\right)^{2}
$$

with $b$ the OTF's sampling pitch. Using these equations, the selected CSIG corresponding to our needs presents the following characteristics :

- Number of diffracted orders : $\mathrm{N}=48$

- Radius of selected orders : $\eta^{2}=9425$

- Recommended CSIG period : $a_{0}=388.3 \mu \mathrm{m}$

These parameters correspond to the following profile :

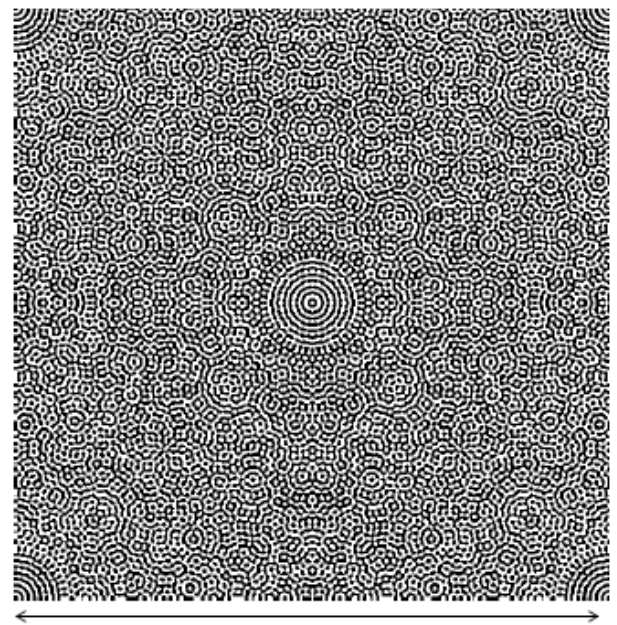

$a_{0}$

Figure 2. Elementary pattern of the recommended 48-orders CSIG approximated by two levels (black and white)

\subsubsection{The translation stages}

The microscan technique implies the use of a two-axis system composed of translation stages allowing a $1 \mu m$ sampled pitch scan. The stages used are SmarAct-2490 piezo-positioners assembled in a X-Y system (cf figure $3)$.

scanning with a $1 \mu \mathrm{m}$ pitch is necessary to not be limited by the pixel cut-off frequency. ${ }^{1}$ However, the positioning errors can reach tens of nanometers for each translation, so it is necessary to minimize these errors as much as possible. The better way to reach this objective is to perform the microscan via a snail "snail" pattern illustrated in figure 4 allowing to do the same moves number following the two axes. 


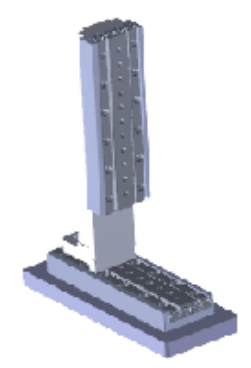

Figure 3. 3D view of the bi-axis system with the smaract piezo-positionners

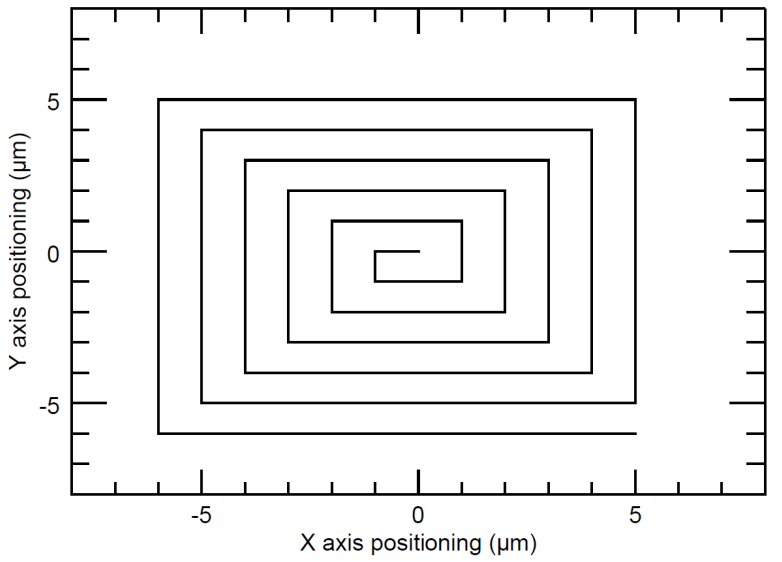

Figure 4. Presentation of the snail course

\subsection{Image processing}

Given an image $i$ of an interferogram delivered by a pixel, $i$ can be modeled as a convolution between $h$ the PSF of the CSIG and $o$ the PSF of the detector. The image is also noisy (additive noise $n$ ). The idea of our method is to operate a deconvolution scheme based on a Bayesian approach, which takes into account the noise in the image and the a priori information on the object to be restored. This data processing method will be presented in the following paragraphs. ${ }^{1}$

The projected interferogram $i$ can be expressed as the convolution of the CSIG's point spread function (PSF) and the pixel's PSF as :

$$
i=h * o+n
$$

The problem is to obtain an estimate of of the observed object $o$ given the image $i$, a precise knowledge of the CSIG's PSF $h$, as well as some prior information on the noise statistics and on the object (the sought detector PSF). Following the Bayesian Maximum a Posteriori (MAP) approach, the deconvolution problem can be stated as follows: we look for the most likely object given the observed image $i$ taking into account prior information on $o$. Figure 5 presents an example of estimated pixel profile with CSIG's PSF. ${ }^{1}$ 

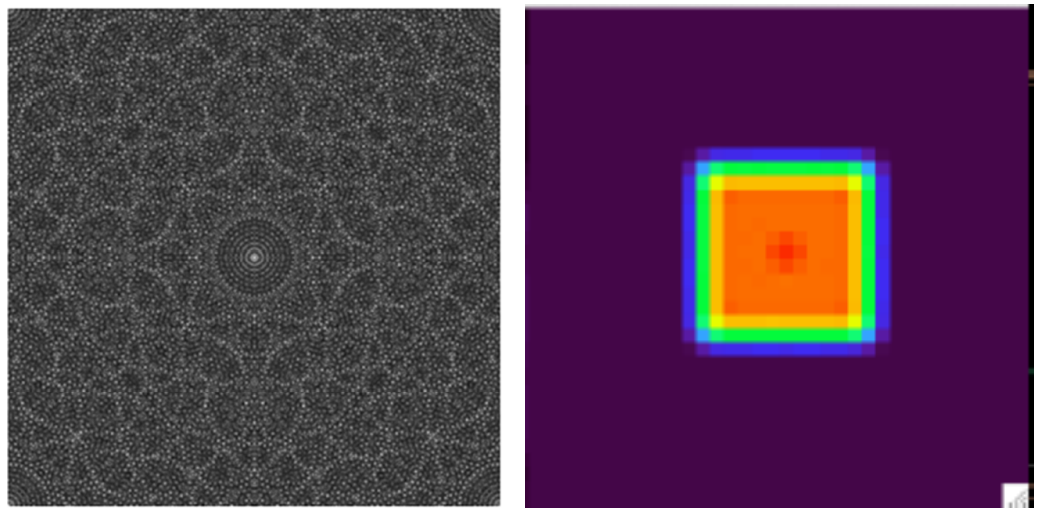

Figure 5. On the left : the CSIG's PSF. On the right : the estimated pixel profile

This method requires a good knowledge of the CSIG's PSF $h$, and a priori on the measurement noise $n .{ }^{9}$ This is why our processing software can be divided in two major parts : a direct model allowing to produce the PSF of the CSIG mounted on the test bench and to simulate the noise sources, and on the second hand the deconvolution part allowing the pixel's PSF reconstruction.

We have used this tool to establish an error budget for the different test bench components.

\section{ESTABLISHMENT OF AN ERROR BUDGET}

Many experimental factors can degrade the pixel estimation. In this paragraph, we will present the main identified contributions to the pixel reconstruction error. Thanks to our direct model, we isolate the different error contributions and study their influence on the reconstruction error. Indeed, as the total error as the quadratic sum of all the error sources, it is firstly necessary to study separately their contributions in order to obtain a relevant error budget. Different contributors have been identified, particularly two major contributors :

- The signal to noise ratio (SNR),

- The translation stages positioning errors.

Other minors contributors have been identified as well :

- The CSIG's orientation,

- The errors due to the test bench vibrations,

In the following paragraphs, we will present the simulation results performed with the direct model for each error contributor.

\subsection{Influence of the SNR}

The signal to noise ratio is defined as the ratio between the signal and the temporal noise standard deviation. To perform these simulations, a Gaussian white noise has been implemented on the simulated interferogram. This noise is generated by a Gaussian distribution centered on the interferogram mean divided by the SNR value to simulate. The simulations have been performed for a SNR varying from 100 to 500 . The evolution curve obtained allows now to predict the error as a function of the experimental SNR :

The simulation results show a very fast degradation of the pixel reconstruction when the SNR decreases. Indeed, whereas a SNR of 500 corresponds to an error of $2 \%$, the curve shows this error goes up to $15 \%$ for an SNR equal to 100. Considering the only SNR contribution, maintaining an error of $10 \%$ would be equivalent to a SNR value of 150. However, it is necessary to keep a margin for the other contributors. This is why the specified SNR for experimental tests is fixed to 500 . 


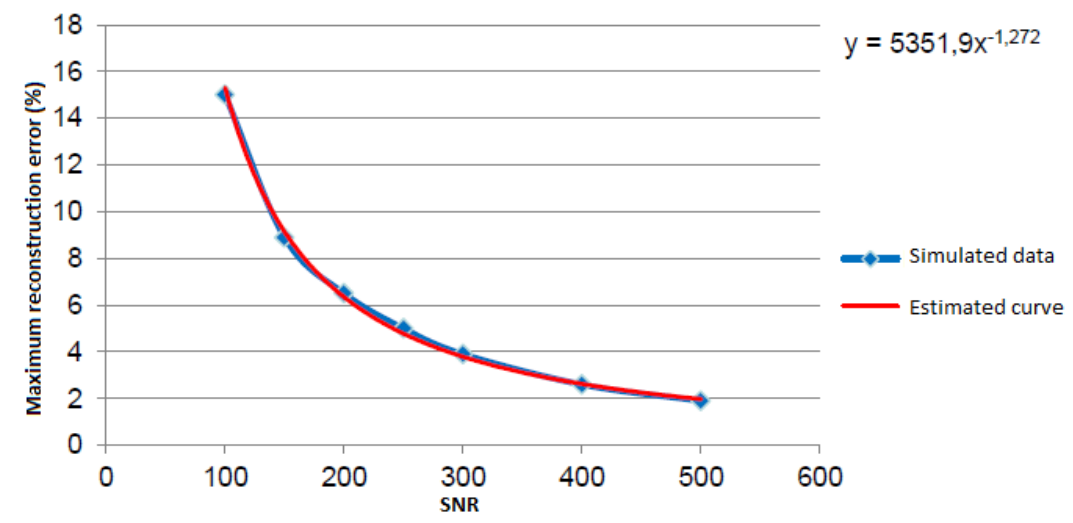

Figure 6. Evolution curve of the SNR contribution to the global error

\subsection{Shifts on the microscan positioners}

The simulation of positioning errors has been considered from an inverse approach: we first generate a noiseless interferogram correctly sampled (pitch $1 \mu \mathrm{m}$ ) with the direct model. The microscan in then performed using a numeric pixels grid sampled at 1 to 12 pixels (which corresponds to our detector sampling), in order to obtain an under sampled cube image. The numerical grid, instead of being translated pixel to pixel, has been translated of a pixel plus a random error of a few tens of nanometers. This error is picked on a Gaussian distribution centered on zero with a standard deviation varying from 0 to $170 \mathrm{~nm}$. The results are presented figure 7 :

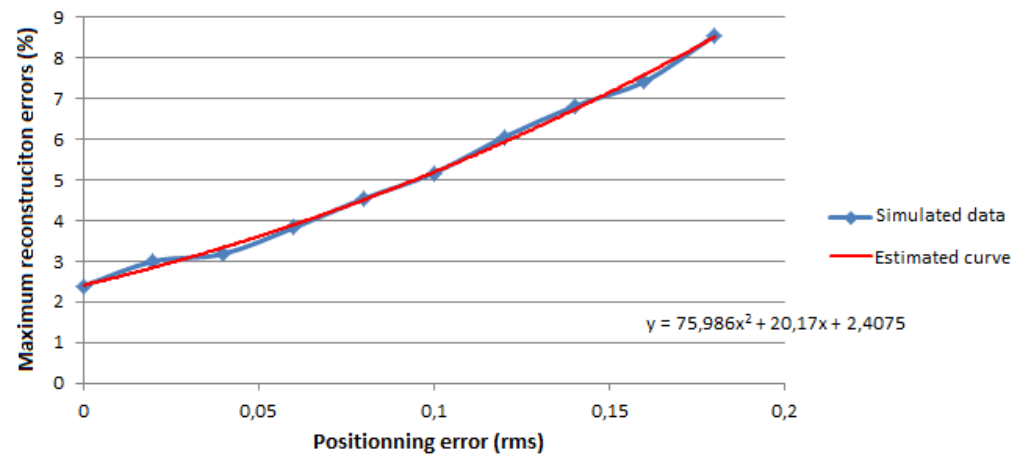

Figure 7. Evolution curve of the shift contribution to the global error

It is interesting to note that a zero shift implies a reconstruction error superior to $2 \%$. As well as the simulations performed for the SNR error contribution, we can note a quick degradation of pixel profile reconstruction performed by our algorithm. Nevertheless, even if the critic value of $10 \%$ is never reached, it is important to minimize as much as possible the experimental shift in order to obtain a reconstruction error bellow the $10 \%$ threshold. Considering the translation stages accuracy, we specify an experimental maximum shift of 150 to 200 $n m$ standard deviation on the whole acquisition cube.

\subsection{CSIG orientation}

CSIG's orientation, which is defined as the angle $\theta$ formed between the grating and the detector pixels columns, is a parameter taken into account in our direct model. It is indeed possible to generate a CSIG PSF with a non-zero angle. This capability has been developed considering that the CSIG experimental angle is not perfectly fixed to zero. We performed reconstruction tests considering the CSIG orientation with angle from 0 to $45 \circ$, angle beyond which the pattern symmetry effects reproduce the same error values. We observe that the reconstruction error does not exceed $2.7 \%$, which remains small comparing to the precedent contributors. 


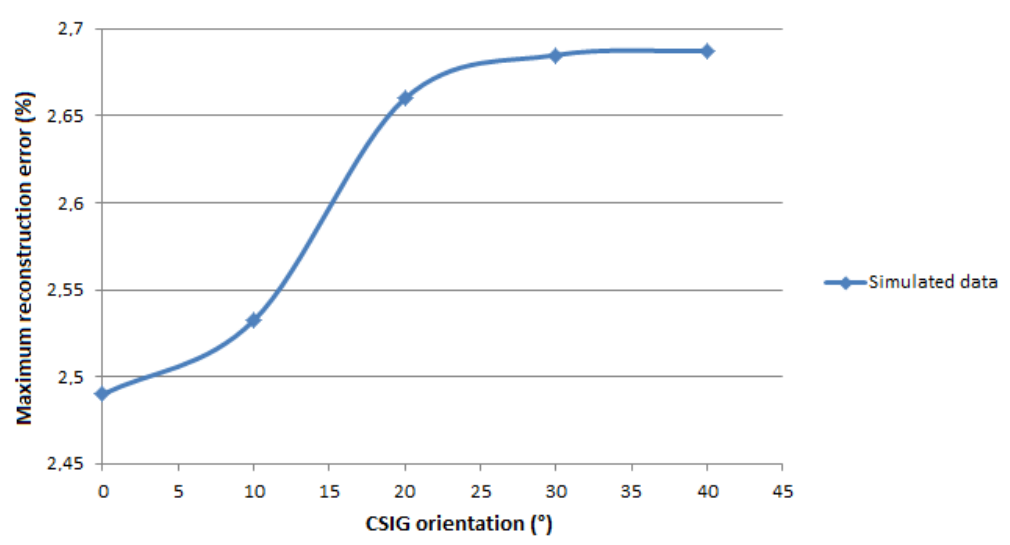

Figure 8. Evolution curve of the CSIG orientation contribution to the global error

This test has been performed for a noiseless interferogram with a perfect microscan.

\subsection{Errors due to the test bench vibrations}

Vibrations can be analyzed from two points of view: a short exposure time and a long exposure time point of view. With a short exposure time, the result will be shifted for the same position of translation stages. In our test bench, this is equivalent to a difference of positioning between the position sent to the stages and the estimated position. This question has already been treated previously (cf. 3.2). With a long exposure time, it is interesting to average several images (in order to improve the SNR for example). The vibration effect is equivalent to a convolution between the interferogram and a circle of radius corresponding to the vibration amplitude.

The simulation has been performed with a noiseless interferogram without simulated microscan. The vibration amplitude varies from 0.5 to $1.5 \mu \mathrm{m}$. The results are presented in figure 9 :

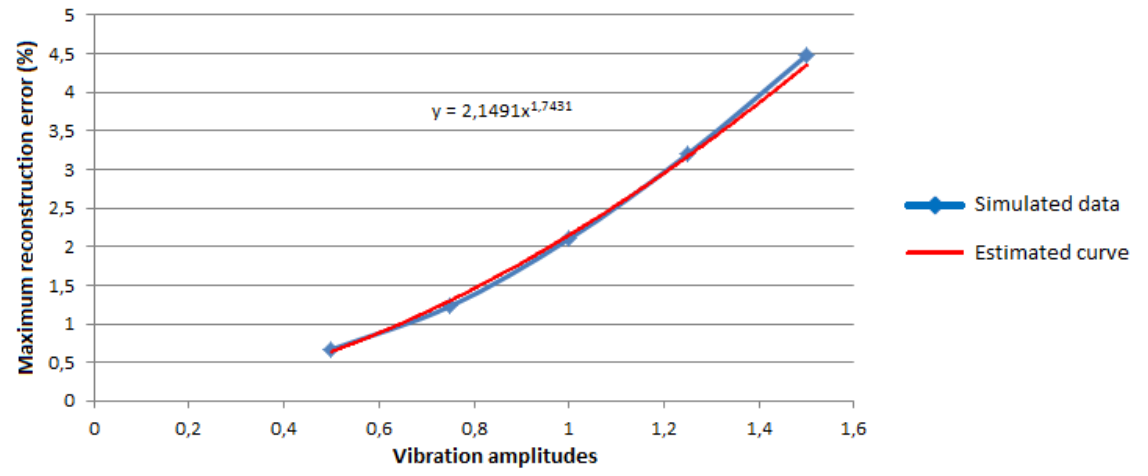

Figure 9. Evolution curve of the vibration amplitude contribution to the global error

The reconstruction error does not exceed 5\%. Considering that the chosen amplitude corresponds to high levels, the specified value will be $0.5 \mu \mathrm{m}$ or less. Indeed, regarding equation 4 , the size of the pinhole in the image plane is $2 \mu \mathrm{m}$. Having an equivalent diameter of $0.5 \mu \mathrm{m}$ would increase of $25 \%$ the total pinhole diameter, which is significant. Nevertheless, the contribution of this parameter to the global error being weak, we can over-estimate it in our global error budget without important consequences.

\subsection{Conclusion on the error budget}

The error budget performed on this test bench allows to isolate the different reconstruction pixel error contributors. Assuming a very good knowledge of the CSIG's orientation, our deconvolution algorithm present a 
sufficient stability allowing a determination of the pixel profile with a accuracy lower than $10 \%$. The next step is to experimentally measure the value of the different contributors in order to provide an estimation of our pixel reconstruction.

\section{FIRST EXPERIMENTAL RESULTS}

The first experimental tests consists in checking the reconstruction error contributors corresponding to the specifications issues of the tolerancing simulations. We will firstly present the experimental values of the error contributors with their measurement methodology, then we will present the first experimental results for our DALSA 1M60 camera tested detector.

\subsection{Signal to noise ratio measurement}

Is this section, we will concentrate on the SNR corresponding to the temporal noise, the spatial noise being considered as negligible for the employed CCD detector.

Experimentally, we consider the SNR as the ratio between the mean signal on the whole matrix and the temporal noise standard deviation for 100 images. The protocol consisted in acquiring an under sampled image of the interferogram (so without microscan) and a noise map for which each pixel $\left(x_{1}, y_{1}\right)$ represents the standard deviation measured to the position $\left(x_{1}, y_{1}\right)$ for 100 images. The exposure time corresponds to the maximum integration time of the camera (60 fps). The image and the noise map are presented in figure 10.
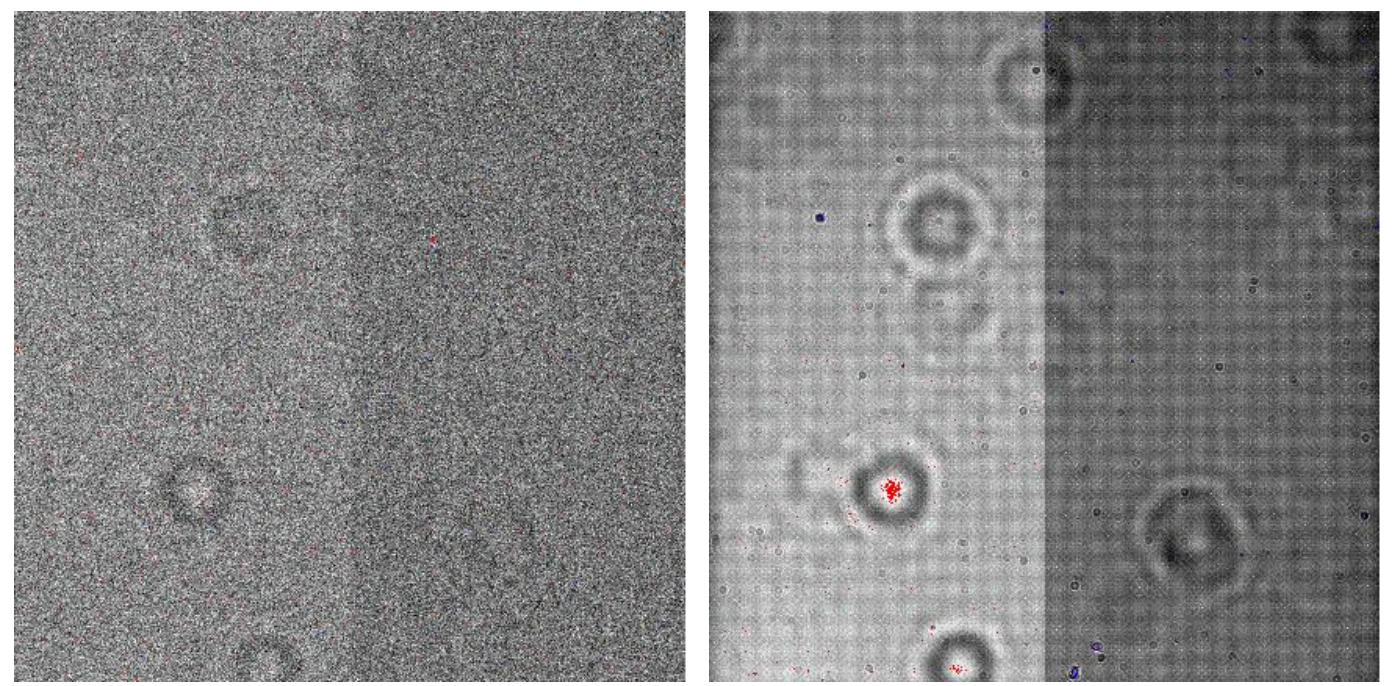

Figure 10. On the left : noise map estimated from 100 images. On the right : non corrected interferogram

These images are acquired without flat and dark corrections. The two different contrast zones correspond to the two CCD outputs. The measured value in these experimental conditions is 80 , which is insufficient to correspond to the previous defined specifications. Rather than increasing the fiber diameter, which could have a filtering effect on the interferogram, it is preferable to apply an averaging of $N_{i} m$ images for each stages positioning. The SNR increasing as $\sqrt{N}$, we chose to perform an averaging for 100 images, which corresponds to an final SNR value of 800 , much higher that the specified performances with the simulations.

from the estimated curve figure 6 , we deduce that the expected reconstruction error is $1.1 \%$.

\subsection{Shifts positioning}

We define the positioning shifts as the gap in $\mu m$ between the wished positioning and the estimated one for the 2 translation axes. In order to estimate these subpixel shifts, it is necessary to use the experimental interferogram phase. Indeed, the phase can be expressed as a first degree polynomial as : 


$$
\phi=2 \pi\left(f_{x_{0}} x_{0}+f_{y_{0}} y_{0}\right)
$$

with $f_{x_{0}}$ the spatial frequencies vector following the $\mathrm{x}$ axis in the Fourier space, $x_{0}$ the interferogram position on the $\mathrm{x}$ axis, $f_{y_{0}}$ the spatial frequencies vector following the $\mathrm{y}$ axis in the Fourier space, $y_{0}$ the interferogram position on the $\mathrm{y}$ axis.

This polynomial phase is estimated with a mean square estimator, which estimates the polynomial coefficients $x_{0}$ and $y_{0}$ corresponding to the interferogram position. The results of this estimation is given in figure 11.
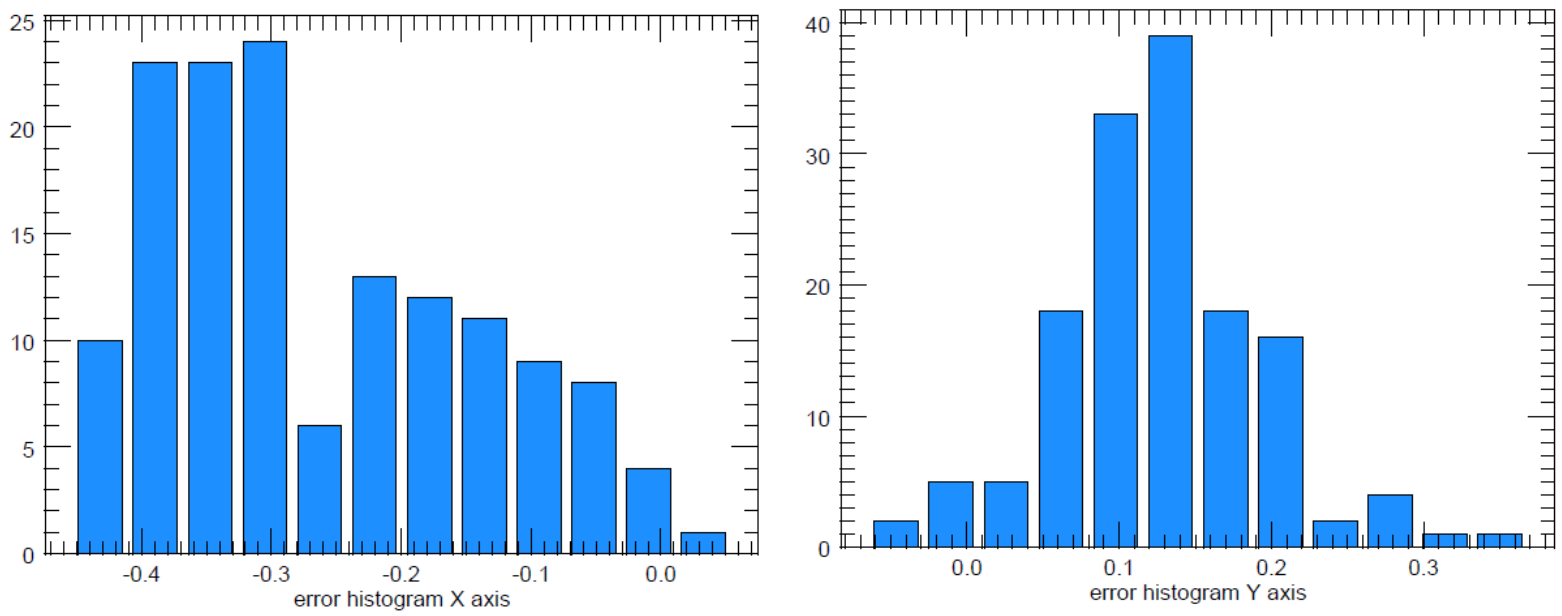

Figure 11. Histogram representing the estimated shifts for the $\mathrm{x}$ and $\mathrm{y}$ axes

Experimental statistics give standard deviations of $152 \mathrm{~nm}$ for the $\mathrm{x}$ axis and $186 \mathrm{~nm}$ for the $\mathrm{y}$ axis, which corresponds to our specifications from our previous simulations. The contribution error expected for these shifts corresponds to $7.22 \%$ for the $\mathrm{x}$ axis and $8.78 \%$ for the $\mathrm{y}$ axis, for a average error of $7.99 \%$.

\subsection{CSIG orientation}

The CSIG orientation is estimated with a multi-parametric estimation algorithm based on a Levenberg Marquardt method. A parametric model generates an under-sampled image with parameters estimating the experimental values of the CSIG period $a_{0}$, the $\theta$ orientation angle of the CSIG, and the position of the interferogram $x_{0}$ and $y_{0}$. The principle of the estimation algorithm is to find the set of parameters $a_{0}, \theta, x_{0}, y_{0}$ which maximizes the similarity between the model and the experiment result. A detailed description of this algorithm can be find in. ${ }^{10}$

The experimental value estimated for $\theta$ is -0.7 ○. This value, even if it is weak, has to be implemented in our direct model in order to generate a PSF with the correct angle.

\subsection{Test bench vibrations}

To estimate vibrations we use the same methodology as to estimate the positioning shifts. The main difference is that we use a set of images for each stages position. The SNR does not need to be as high as for the pixel's reconstruction, this is why the images are acquired without averaging, which will allow to determine the long exposure time effect we described previously. The experimental results are presented figure 12 .

The experimental measured vibrations have a maximum amplitude of $0.1 \mu \mathrm{m}$, which corresponds according to the evolution curve obtained with our simulations to a reconstruction error of $0.03 \%$ cf. figure 9 . This is above the specifications we fixed with our simulation tests, however the contribution to the global error remains small. 


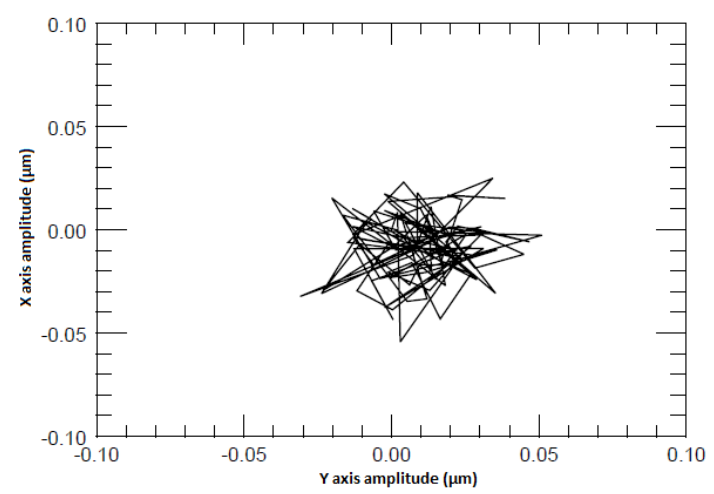

Figure 12. Vibration amplitude measured on the test bench

\subsection{Estimated pixel profile}

According to the contributions of the different test bench components in the global reconstruction error, the pixel profile will be estimated with an error of $8.5 \%$, corresponding to the quadratic sum of the different contributions determined previously.

In order to reduce the acquisition time, we proceed to an estimation of an average pixel on a zone of $100 \times 100$ pixels of the detector.

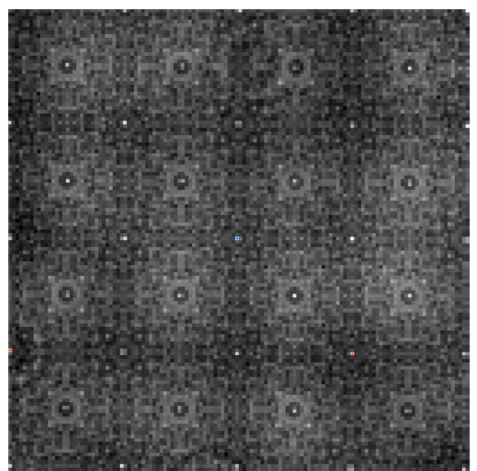

Figure 13. Under sampled interferogram image for a 100x100 pixels zone, before micro-scanning

The micro-scanning step over-samples the interferogram, which results in the convolution of the detector's PSF with the CSIG's PSF :

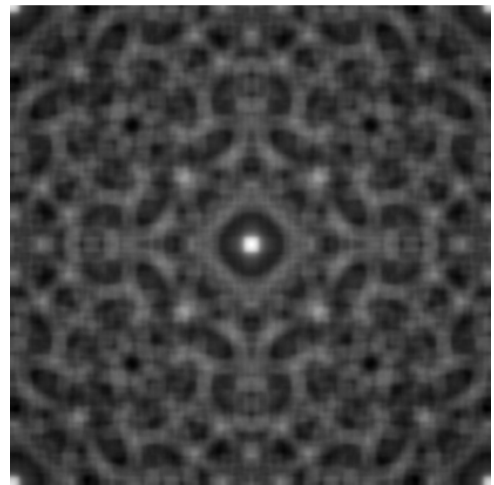

Figure 14. Over-sampled interferogram reconstructed after microscan

The pixel reconstructed by the deconvolution software presents a square profile for a square size of $12 \mathrm{x} 12$ 
$\mu m$, which corresponds to the data given by the manufacturer. The pixel support is $48 x 48 \mu m^{2}$. Moreover, the sectional view shows an offset for the estimated pixel. Although an improvement has been noted on the estimated pixels artifacts, comparing to the precedent paper, ${ }^{1}$ we can see a residual offset on the reconstructed pixel thanks to the sectional view. This shows a certain stability of the result of our deconvolution process. These residual artifacts can be seen as a lack of parameters in the direct model which prevents the generation of a perfect PSF. Many reflexions are under study in order to improve the direct model and remove this residual offset.

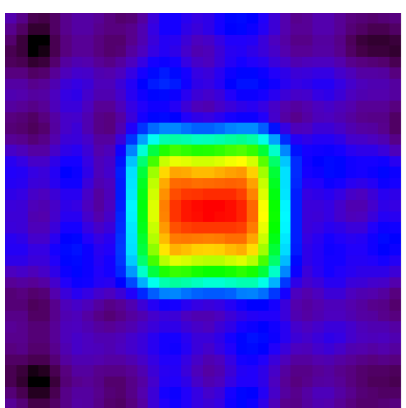

Figure 15. 2D view of the reconstructed pixel

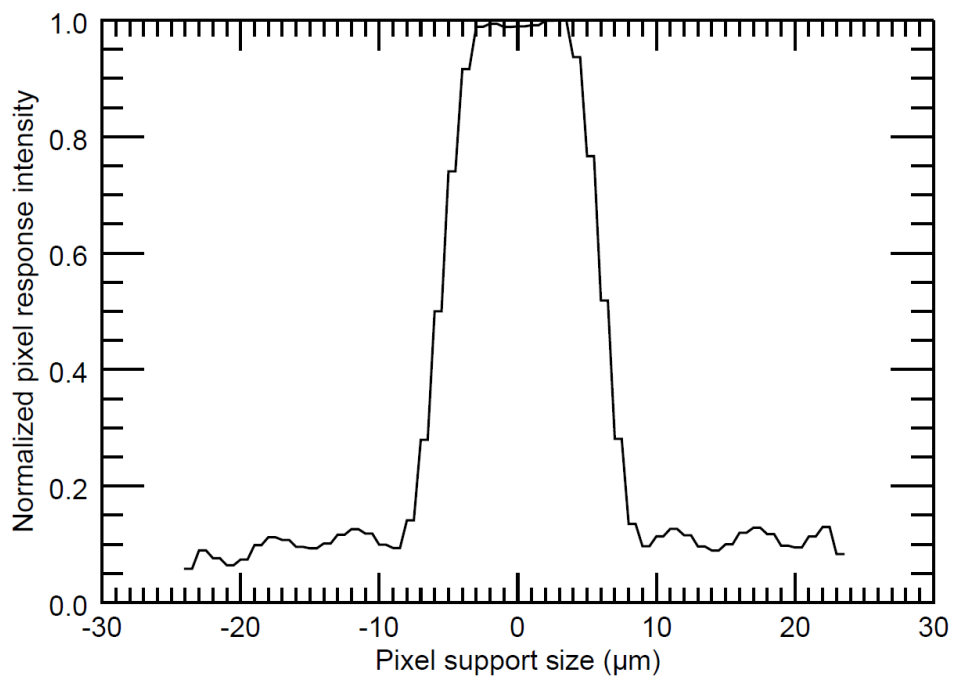

Figure 16. Sectionnal view of the reconstructed pixel 


\section{ONGOING DEVELOPMENTS AND PERSPECTIVES}

The final objective of this work is to design a versatile test bench which will allow to carry the measurements in different spectral bands going from the visible (VIS) to the long-wave infrared (LWIR, up to $13 \mu m$ ). The constraints of operation in the LWIR drive us to mount the collimator, the detector and the CSIG inside a cryostat. Figure 17 below gives a synopsis of the final INTRAPIX test bench.

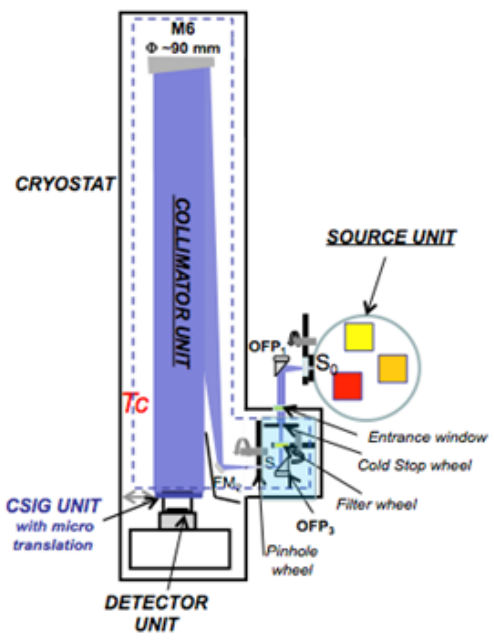

Figure 17. Schematic representation of the INTRAPIX bench and its different functional units. See text for detailed description.

Following the pathway of the light, we have identified 5 functional units: a Source unit which consists in a light generator and an assembly of mirrors to collect the light and reflect it towards the entrance window of the cryostat. Inside the Cryostat unit, the light encounters a pre-optics stage which re-images the point source S0 at the focus of the Collimator unit (the point $S$ in the picture). For compactness purpose, the Collimator unit is a two-folded system (see figure 17) and produces a parallel beam that impinges the CSIG unit. And finally the light diffracts and recombines to produce a self-image of the grating on the detector, the main component of the Detector unit.

The choice of a unique test bench to cover several bands in the spectrum $(0.4$ to $13 \mu \mathrm{m})$ imposes to design each unit with high attention. The main driving idea is to reduce as much as possible the modifications of the setup when we move from one band to another. The architecture choices are then sustained by the aim to fill the following objectives together: limitation of wavefront error at the exit of the collimator under $\lambda / 10$ RMS (at 633 $\mathrm{nm}$ ), reduction of the thermal background contribution below the light generator photon noise, improvement of the system mechanical stability and limitation of the amount of the diffused light.

To limit the thermal background, the Collimator and the CSIG are mounted inside the cryostat and cooled to $40 \mathrm{~K}$. The Source unit is located outside the cryostat to avoid spurious light and also to limit the mas to be cooled. The refrigeration of the environment will be achieved with a PT415 cryorefrigerator from Cryomech Inc, a two-stage pulse-tube cooler. The first stage has $40 \mathrm{~W}$ cooling capacity at $45 \mathrm{~K}$ and the second has $1.5 \mathrm{~W}$ of cooling power at $4.2 \mathrm{~K}$. The second stage referred as $4 \mathrm{~K}$-stage, will be dedicated for the cooling of the detector which is also mounted inside the cryostat while the 45K-stage will be dedicated to the Collimator and the CSIG units. Specific flexible thermal links will be designed to connect the cold stages to the different units.

The Collimator unit consists mainly in 2 plane mirrors, 2 parabolic off-axis mirrors, 2 wheels (one for spectral filters and the other for pinholes), and the optical bench on which the previous elements are attached once and for all. The bench, the mirrors and their mounting will be realized from materials with very similar thermal expansion coefficients in order to obtain homogeneity of the shrinking during the cooling down and cooling up. 
Thereby no re-alignment will be required after a temperature variation indeed as the distance and power of the mirrors change in a homogeneous manner, the pinhole remains at the focus of the Collimator unit. All the mirrors shall be made from Rapid Solidified Aluminum 6061 (RSA 6061). This alloy has fine nano-structures and gives an optical surface characterized by very small roughness values in few diamond machining steps. It offers the potential to reach a final roughness better than $4 \mathrm{~nm}$ RMS. Under this threshold, the amount of diffused light in the VIS case should be negligible.

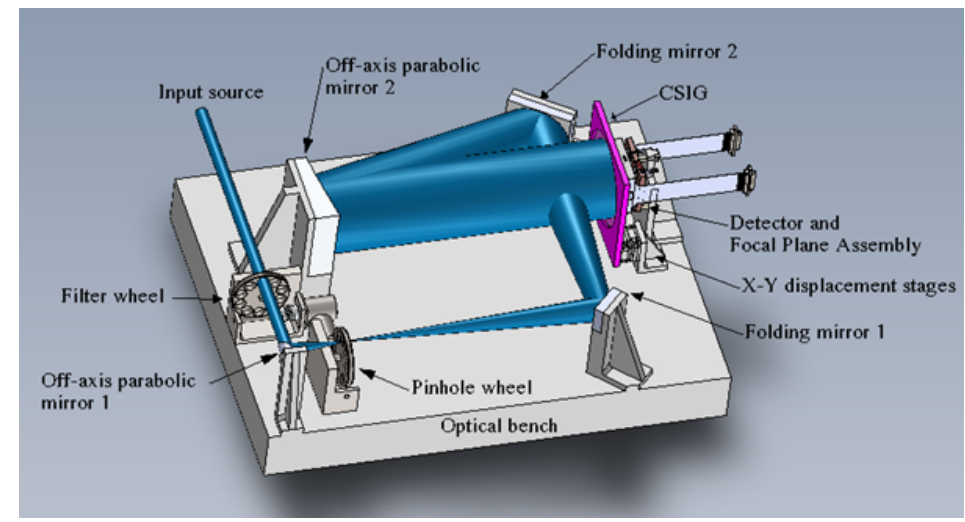

Figure 18. Illustration of the Collimator Unit, the CSIG and the Detector. The Source unit and the Pulse Tube are not represented.

The CSIG unit contains the grating and its holder. Three gratings have been manufactured by the SILIOS company, to cover the VIS, NIR and LWIR bands. During the detector characterization phase in a given band, the appropriated grating shall be mounted at $10 \mathrm{~mm}$ from the detector and used under normal incidence. The gratings permit to explore the spatial response at a frequency several times higher than the Nyquist frequency defined by the detector as an image sampler. To get rid of aliasing effect, a super-resolution procedure will be implemented as in the demonstrator case. To ensure these displacements in the cryogenic environment, 2 piezo nano-positioners (ANP Series, Attocube) will be used in closed loop control based on fiber-optic Fabry-Perot interferometers (FPS, Attocube) The interferometer is not represented in figure 18. The accuracy achieved by this combination is better than $20 \mathrm{~nm}$ for $1 \mu \mathrm{m}$ displacement step namely 10 times better than the performance achieved with the demonstrator. Using optical fibers for the interferometers as close to the detector demands to design suitable baffling to avoid spurious light hitting the sensitive area of the DUT (Device Under Test).

The Detector unit contains the detector to test and the focal plane assembly (FPA). The FPA has different interfaces (mechanical, electrical and thermal) and it will be designed as versatile as possible to be able to receive different detectors without major additional developments. The first detectors we expect to characterize in the INTRAPIX test bench are the following:

- CCD-273, e2v, Back illuminated, deep depletion and red-enhanced response, full frame, 4132x4096 pixels, $12 \mu \mathrm{m}$ pitch. This device is the detector that will populate the visible channel of the Euclid payload - NIR Photodiodes, CEA Leti/Sofradir, MCT technology, Cut-off @ $2.1 \mu m, 2048 \times 2048$ pixels, $15 \mu m$ pitch - LWIR Photodiodes, CEA Leti/Sofradir, MCT technology, Cut-off @ $12.5 \mu \mathrm{m}, 640 x 512$ pixels, $15 \mu \mathrm{m}$ pitch - AQUARIUS, Raytheon, Si:AC Impurity Band Conduction, range: [3-28] $\mu m, 1024 x 1024$ pixels, $30 \mu m$ pitch. This device is one of the two types of science detectors of the E-ELT METIS instrument.

To conclude this section, the table below summarizes the detectors features and the level of knowledge of the pixel PSF we expect to reach with the INTRAPIX test bench: 


\begin{tabular}{|c|c||c||c|c|}
\hline $\begin{array}{c}\text { Spectral } \\
\text { characteristics }\end{array}$ & VS & NIR & \multicolumn{2}{|c|}{ LWIR } \\
\hline $\begin{array}{c}\text { Spectral band } \\
(\mu \mathrm{m})\end{array}$ & $0.5-0.9$ & $1.0-1.8$ & \multicolumn{2}{|c|}{$8.4-13$} \\
\hline \hline Detectors & $\begin{array}{c}\text { CCD-204-22 } \\
\text { CCD-273 }\end{array}$ & NIR LFSA Léti & AQUARIUS & LWIR Léti \\
\hline \hline Pixel $(\mu \mathrm{m})$ & 12 & 15 & 30 & 15 \\
\hline $\begin{array}{c}\text { Number of } \\
\text { pixols }\end{array}$ & $4096 \times 4132$ & $2048 \times 2048$ & $1024 \times 1024$ & $640 \times 512$ \\
\hline \hline Size $(\mathrm{mm})$ & $49.152 \times 49.584$ & $30.72 \times 30.72$ & $30.72 \times 30.72$ & $9.6 \times 7.68$ \\
\hline \hline $\begin{array}{c}\text { Resolution } \\
\text { (pixel) }\end{array}$ & $1 / 12$ & $1 / 12$ & $1 / 4$ & $1 / 5,1 / 4$ \\
\hline $\begin{array}{c}\text { Temperature of } \\
\text { dotector }\end{array}$ & $150-160 \mathrm{~K}$ & $80-100 \mathrm{~K}$ & $5-7 \mathrm{~K}$ & $30-50 \mathrm{~K}$ \\
\hline \hline
\end{tabular}

Figure 19. Detector features and the resolution expected in the pixel PSF measurement

\section{CONCLUSION}

In this paper, we have presented the first results and demonstrated the experimental feasibility of our test bench. Our approach, based on a simple projection technique, allows to explore the pixel response of a CCD detector with a very high accuracy. The error budget determined allows to estimate a global error on our measurement lower than $10 \%$, with a resolution to the twelfth of pixel. Ongoing developments are preparing the definitive version of the test bench for several spectral bandwidths. Although we have already reduced the artifact effects and improved the deconvolution process, future works need to improve the direct model in order to reduce to zero the residual offset on the estimated pixel.

\section{ACKNOWLEDGMENTS}

This work has been partially supported by the LabEx FOCUS ANR-11-LBX-0013. The development of the final test bench is supported by the DIM-ACAV and the LabEx P2IO.

\section{REFERENCES}

[1] Ketchazo, C., Viale, T., Boulade, O., Druart, G., Moreau, V., Mugnier, L., Dubreuil, D., Derelle, S., Ronayette, S., Guérineau, N., and Berthe, M., "A new technique of characterization of the intrapixel response of astronomical detectors," Proc. SPIE 9154, 91541Y-91541Y-14 (2014).

[2] Lauer, T. R., "The photometry of undersampled point spread functions," Publications of the Astronomical Society of the pacific 111(765), 1434-1443 (1999).

[3] Laureijs, J. R., "Euclid definition study report," tech. rep. (2011).

[4] Guérineau, N., Primot, J., Tauvy, M., and Caes, M., "Modulation transfer function measurement of an infrared focal plane array by use of the self-imaging property of a canted periodic target," Appl. Opt. 38, 631-637 (Feb 1999).

[5] Guérineau, N., Harchaoui, B., Primot, J., and Heggarty, K., "Generation of achromatic and propagationinvariant spot arrays by use of continuously self-imaging gratings," Opt. Lett. 26, 411-413 (Apr 2001).

[6] Niemi, S.-M., Cropper, M., Szafraniec, M., and Kitching, T., "Measuring a charge-coupled device point spread function," Experimental Astronomy 39(2), 207-231 (2015).

[7] Swindells, I., Wheeler, R., Darby, S., Bowring, S., Burt, D., Bell, R., Duvet, L., Walton, D., and Cole, R., "Mtf and psf measurements of the ccd273-84 detector for the euclid visible channel," Proc. SPIE 9143, 91432V-91432V-8 (2014).

[8] Piponnier, M., Druart, G., Guérineau, N., de Bougrenet, J.-L., and Primot, J., "Optimal conditions for using the binary approximation of continuously self-imaging gratings," Opt. Express 19, 23054-23066 (Nov 2011).

[9] L. M. Mugnier, T. F. and Conan, J.-M., "Mistral: a myopic edge-preserving image restoration method, with application to astronomical adaptive-optics-corrected long-exposure images," J. Opt. Soc. Am. A 21, 1841-1854 (Oct 2004).

[10] de la Barrière, F., Druart, G., Guérineau, N., Taboury, J., Primot, J., and Deschamps, J., "Modulation transfer function measurement of a multichannel optical system," Appl. Opt. 49, 2879-2890 (May 2010). 